\title{
Evaluation of plasma levels of neopterin and soluble CD40 ligand in patients with acute ischemic stroke in upper Egypt: can they
} surrogate the severity and functional outcome?

This article was published in the following Dove Medical Press journal:

Neuropsychiatric Disease and Treatment

\author{
Abeer A Tony' \\ Effat AE Tony ${ }^{2}$ \\ Wafaa Salah Mohammed ${ }^{3}$ \\ Emad F Kholef ${ }^{3}$ \\ 'Department of Neuropsychiatry, \\ Faculty of Medicine, Aswan University, \\ Aswan, Egypt; ${ }^{2}$ Department of Internal \\ Medicine, Nephrology Unit, Faculty \\ of Medicine, Assuit University, Assuit, \\ Egypt; ${ }^{3}$ Department of Clinical \\ Pathology, Faculty of Medicine, Aswan \\ University, Aswan, Egypt
}

Background: Inflammation constitutes a major component of ischemic stroke pathology. The prognostic value of "neopterin" and soluble CD40 ligand (sCD40L), as a potential biomarker of ischemic stroke, has been less extensively studied.

Objectives of the study: This study aims at assessing the serum levels of neopterin and sCD40L in acute ischemic stroke (AIS), to clarify its association with the severity, etiology, and risk factors of stroke, and to evaluate their relationship with the stroke functional outcome in our study sample within 90 days of follow-up.

Study sample: This case-control study was conducted on 100 patients with first-ever acute onset ischemic stroke and 25 control subjects.

Methods: Participants were subjected to full history taking and detailed clinical and neurological examination. Brain imaging was performed after hospital admission. Blood tests were drawn for assessment of neopterin and $\mathrm{SCD} 40 \mathrm{~L}$ on the first day of admission.

Results: High levels of neopterin and sCD40L was reported. Their levels were significantly higher in relation with survival status. There was a relationship between AIS and SCD40L levels and the severity of the stroke. Within 3 months of follow-up, these biomarkers were associated significantly with poor functional outcome, within a 90-day follow-up period, and mortality. These biomarkers were highly associated in patients with small vessel occlusion as an etiology for AIS.

Conclusion: Neopterin and sCD40L levels increased after AIS. Both biomarkers were strong and independent predictors of 90-day unfavorable clinical outcome and death in patients after AIS. Keywords: acute ischemic stroke, soluble CD40 ligand, neopterin, stroke risk factors, functional outcome

\section{Introduction}

Ischemic stroke accounts for the majority of stroke cases and constitutes a major cause of death and disability in the industrial world. ${ }^{1}$ Inflammation and immune response play an important role in the pathogenesis of ischemic stroke. ${ }^{2,3}$ The brain responds to ischemic injury with an acute and prolonged inflammatory process, characterized by rapid activation of resident cells, production of pro-inflammatory mediators, and infiltration of various types of inflammatory cells into the ischemic brain tissue. ${ }^{4-6}$ Neopterin is a pteridine derivative which is mainly produced by macrophages stimulated by IFN-Y which is secreted by activated T-cell lymphocytes. ${ }^{7}$ It is a marker associated with the activity of monocyte/macrophages ${ }^{8}$ and a sensitive marker of cell-mediated
Correspondence: Abeer A Tony Department of Neuropsychiatry, Faculty of Medicine, Aswan University, Sahary Discrit, Airport street, Aswan 81528, Egypt

Tel +20973480901

Fax +20973480449

Email abeer.tony@aswu.edu.eg 
immunity. ${ }^{9}$ Neopterin is also formed within the central nervous system by the microglia cells. ${ }^{10}$ It can be detected in various body fluids such as serum, cerebrospinal fluid, synovial fluid, pancreatic juice, urine, saliva, and ascites fluid. ${ }^{11,12}$ Neopterin concentrations are found to be increased in atherosclerosis, infectious diseases, malignancies, autoimmune diseases, insulin-dependent diabetes mellitus, heart and kidney failure, coronary artery disease, cerebral ischemia, multiple sclerosis, and allograft rejection. ${ }^{2}$ Zeng et al stated that early increases in serum level of neopterin can predict 90-day unfavorable clinical outcome in patients after AIS. ${ }^{2}$ Therefore, it can be used as a useful independent tool to predict functional outcome and mortality 6 months after stroke. Long-term disability is a significant problem among survivors, but much of the literature has emphasized mortality rates as an outcome measure rather than functional status and reduction of morbidity. ${ }^{13}$ CD40L (CD40 ligand) and sCD40L (soluble form of CD40 ligand) are molecules with a dual pro-thrombotic and pro-inflammatory roles. However, the exact mechanisms linking the CD40/CD40L system and sCD40L with atherothrombosis are currently a topic of intensive research. ${ }^{14} \mathrm{CD} 40 \mathrm{~L}$ is a $39 \mathrm{KDa}$ trimeric transmembrane glycoprotein belonging to the tumor necrosis factor family, which rapidly appears on the surface of the membrane after platelet activation and generates a soluble form, sCD40L. ${ }^{15}$ The levels of sCD40L were reported to increase in patients with cerebrovascular disease and were considered to participate in the process of forming and extending atherosclerosis, including exacerbating the rupture of atherosclerotic plaque. ${ }^{16,17}$ There is a growing evidence that $\mathrm{C}$-reactive protein (CRP), a peripheral marker of inflammation, is also a marker of generalized atherosclerosis ${ }^{18}$ and is a potential prognostic biomarker of ischemic stroke. ${ }^{19}$ VanGilder et al suggested that CRP is a biomarker for predicting post-ischemic stroke outcome. ${ }^{13}$ Moreover, Elkind et al showed an association between high CRP level and stroke presentation, outcomes, and future vascular events. ${ }^{20}$ The current study aimed at evaluating the relationship of neopterin and sCD40L levels with acute ischemic stroke (AIS), clarifying its association with the severity, etiology, and risk factors, and finally assessing their relationship with the stroke functional outcome in our studied patients within 90-day follow-up period.

\section{Patients and methods}

\section{Study design}

This work was a case-control study conducted on 100 patients with first-ever acute onset ischemic stroke and 25 age- and sex-matched apparently healthy subjects as normal controls.

\section{Case selection}

The patients' age ranged from 38 to 80 years with a mean of 60.5 years, and were recruited from The Stroke Unit of Neuropsychiatric Department, Aswan University Hospitals, during the period from September 2015 to September 2016.

1. Inclusion criteria: patients were eligible for study if they were admitted to the stroke unit with ischemic stroke and symptom onset within 24-48 hours, confirmed by computerized tomography (CT scan) and/or magnetic resonance imaging (MRI) of brain.

2. Exclusion criteria: patients with brain neoplasm, history of previous stroke, hepatic/renal impairment, autoimmune diseases, history of acute/chronic inflammatory diseases, malignancy, trauma, surgery, and acute vascular diseases that occurred within 4 weeks prior to the onset of stroke.

\section{Study tools}

All patients were submitted for the following evaluation:

1. Complete neurological examination where the following clinical and demographical data were collected: age, sex, and presence of stroke risk factors (as smoking history, hyperlipidemia, diabetes mellitus, history of hypertension, history of transient ischemic attacks, history of myocardial infarction, or any cardiac problems).

2. Stroke etiology was classified according to the criteria of Trial of Org 10172 in Acute Stroke Treatment (TOAST) classification.

3. Stroke severity was assessed by Scandinavian Stroke Scale (SSS) measured at the time of admission.

4. The modified Rankin Scale (mRS), being one of the most widely used measures for assessment of the outcome of stroke, was used to assess functional outcome at 3 months (90 days after admission) and the patients were blinded to neopterin and sCD40L levels. A mRS $\leq 2$ was defined as favorable outcome, and a $\mathrm{mRS}>2$ was defined as unfavorable outcome. Poor outcome was defined as a score of more than 2 on the mRS, including death at 3 months from stroke onset. A secondary outcome was death at 3 months.

5. Brain imaging (either CT scan and/or MRI) was performed after admission.

6. Electrocardiography and echocardiography were conducted for all patients.

7. Ten milliliters of venous blood was collected from all the studied patients and controls: $2 \mathrm{~mL}$ was drawn into an EDTA tube for complete blood picture (Sysmex $\mathrm{XP}-300$ cell counter) and $8 \mathrm{~mL}$ was divided into two clean tubes (for serum sample collection) for routine and 
special parameter estimation. Serum samples were left for clotting, and then were centrifuged at 3,000 rpm for 20 minutes. Serum was separated and divided into three portions: one for routine parameter estimation (random blood glucose, renal and liver function tests, and lipid profile). All the tests were performed on fully automated analyzer BT-3500 (Biotecnica S.p.A, Rome, Italy). The other two portions were freezed at $-20^{\circ} \mathrm{C}$ until further use for estimation of neopterin and sCD40 ligand levels using a sandwich ELISA technique (Calbiotech Inc., 10461 Austin Dr, Spring Valley, CA, USA). All blood samples were collected on the first day of admission and hemolyzed samples were excluded. Serum level of highsensitivity CRP was measured at admission.

\section{Sample size calculation}

The sample size was calculated using $\mathrm{G}^{*}$ Power 3 software. A minimum sample of 55 participants divided into two groups (30 stroke patients and 25 controls) was needed to detect an effect size of 0.3 in the mean neopterin level, with an error probability of $0.05 \%$ and $80 \%$ power on a one-tailed test. For better analysis, we increased the patients' sample size to include 100 stroke patients.

\section{Statistical analysis}

The collected data were verified, coded by the researcher, and analyzed using the SPSS software, version 21 (IBM Corporation, Armonk, NY, USA). Descriptive statistics including mean, SD, median, range, frequencies, and percentage were calculated. Student's $t$-test, test of significance, was calculated to test the mean differences in continuous variables between groups. Multivariate logistic regression analysis was performed to investigate the significant factors influencing stroke mortality (OR, 95\% CI). Spearman's rank correlation coefficient was calculated for univariate correlations between markers and risk factors. Receiver operating characteristic (ROC) curve was depicted to explore the diagnostic performance of neopterin, $\mathrm{sCD} 40 \mathrm{~L}$, and CRP for diagnosis of stroke, analyzed as area under the curve (AUC), standard error (SE), and 95\% CI. Validity statistics (sensitivity, specificity) were calculated. Test results were considered significant when $P$-value was $\leq 0.05$.

\section{Ethical consideration}

The current study was approved by the Institutional Ethics Committee, Faculty of Medicine, Aswan University. All patients were provided with complete information about the study objectives, methods, and risk/benefit assessment.
A written consent was obtained from each participant upon acceptance to take part in the study. The study was conducted in accordance with the principles of Declaration of Helsinki.

\section{Results}

A total of 100 patients (44 men, 56 women; mean age, $60.6 \pm 9.7$ years; range, $38-80$ years) were included with mean duration of AIS of $18.9 \pm 10.7$ hours. Demographic characteristics and risk factor profiles are presented in Table 1 . The median SSS score was 20 with a mean \pm SD of 25.3 \pm 14 .8. Likewise, the median $\mathrm{mRS}$ score at 90 days after the onset of accident was 4 with a mean \pm SD of $3.7 \pm 1.5$. Their SBP and DBP were high with mean of $144.8 \pm 17.1$ and $84.8 \pm 7.6 \mathrm{mmhg}$, respectively.

Moreover, it was found that 68 patients had abnormal carotid duplex, while 24 cases had abnormal vertebrobasilar duplex. Also, $60 \%$ of cases had AIS due to small vessel disease, while cardio-embolic etiology was observed only in $40 \%$. Non-valvular atrial fibrillation (AF) was the commonest risk factor (84\%) among patients followed by hypertension (64\%), hyperlipidemia (60\%), smoking (40\%), and diabetes $(36 \%)$, whereas the rate of coronary heart disease was the least $(12 \%)$ in the studied stroke cases.

The current study concluded that cases had higher levels of total cholesterol, low-density lipoprotein cholesterol, highdensity lipoprotein cholesterol, triglycerides, and random blood glucose levels.

With regard to the mean serum levels of inflammatory markers, a statistically significant increase in neopterin, sCD40L, and CRP levels was observed in our studied patients with AIS (mean \pm SD: $16.04 \pm 3.8 \mathrm{nmol} / \mathrm{L}, 8.2 \pm 1.9 \mathrm{ng} / \mathrm{mL}$, and $4.8 \pm 1.6$ $\mathrm{mg} / \mathrm{dL}$, respectively) in comparison to control group (mean $\pm \mathrm{SD}$ : $1.3 \pm 0.2 \mathrm{nmol} / \mathrm{L}, 0.8 \pm 0.6 \mathrm{ng} / \mathrm{mL}$, and $2.7 \pm 1.7 \mathrm{mg} / \mathrm{dL}$, respectively) with $P<0.001$ for each parameter as given in Table 2 .

There was no statistically significant difference between the mean serum levels of neopterin and $\mathrm{SCD} 40 \mathrm{~L}$ in AIS cases with favorable ( $\mathrm{mRS}=0-2$ ) and unfavorable ( $\mathrm{mRS}=3-6)$ outcomes 90 days later (mean \pm SD: $16 \pm 3.6$ vs $16.04 \pm 3.8 \mathrm{nmol} / \mathrm{L}$, respectively, with $P>0.05$ and $8 \pm 0.6$ vs $8.2 \pm 2.1 \mathrm{ng} / \mathrm{mL}$, respectively, with $P>0.05$ ) as given in Table 3 and Figure $1 \mathrm{~A}$ and $\mathrm{B}$.

Furthermore, the studied AIS cases showed statistically significant lower mean levels of neopterin in survivors compared to those who died (mean \pm SD: $16 \pm 3.8$ vs $18 \pm 5.2 \mathrm{nmol} / \mathrm{L}$, respectively, with $P \leq 0.001$ ). Notably, the mean levels of CD40 ligand in survivors were significantly lower compared to those who died (mean \pm SD: $8 \pm 1.35$ vs $10 \pm 2.28 \mathrm{ng} / \mathrm{mL}$, respectively, with $P \leq 0.001$ ) as given in Table 4 and Figure $2 \mathrm{~A}$ and $\mathrm{B}$. 
Table I Basic characteristics of the study patients with acute ischemic stroke

\begin{tabular}{|c|c|c|}
\hline Parameters & Patients $(n=100)$ & Controls $(n=25)$ \\
\hline Age/years (mean \pm SD) & $60.6 \pm 9.7$ & $58.5 \pm 5.7$ \\
\hline Sex (male/female) (\%) & $44 / 56(44 \% / 56 \%)$ & $12 / 13(48 \% / 52 \%)$ \\
\hline Duration of stroke (hours) & $18.9 \pm 10.7$ & \\
\hline mRS ( 90 days later) (mean $\pm S D$, median) & $3.7 \pm 1.5,4$ & \\
\hline SSS (mean \pm SD, median) & $25.3 \pm 14.8,20$ & \\
\hline Number of deaths & $24(24 \%)$ & \\
\hline $\begin{array}{l}\text { Vascular risk factors (No [\%]) } \\
\text { Hypertension } \\
\text { SBP }(\mathrm{mm} \mathrm{Hg}) \\
\text { DBP }(\mathrm{mm} \mathrm{Hg}) \\
\text { Dyslipidemia } \\
\text { Diabetes mellitus } \\
\text { Smoking } \\
\text { Ischemic heart disease } \\
\text { Non-valvular atrial fibrillation }\end{array}$ & $\begin{array}{l}64(64 \%) \\
144.8 \pm 17 \\
84.8 \pm 7.6 \\
60(60 \%) \\
36(36 \%) \\
40(40 \%) \\
12(12 \%) \\
84(84 \%)\end{array}$ & \\
\hline $\begin{array}{l}\text { Stroke etiology (No [\%]) } \\
\text { Small vessel occlusion } \\
\text { Cardio-embolism }\end{array}$ & $\begin{array}{l}60(60 \%) \\
40(40 \%)\end{array}$ & \\
\hline Abnormal carotid duplex (No [\%]) & $68(68 \%)$ & \\
\hline Abnormal vertebrobasilar duplex (No [\%]) & $24(24 \%)$ & \\
\hline Laboratory investigations & Mean \pm SD & \\
\hline $\begin{array}{l}\text { TC }(\mathrm{mmol} / \mathrm{L}) \\
\text { LDL-C (mmol/L) } \\
\text { HDL-C (mmol/L) } \\
\text { TG (mmol/L) } \\
\text { RBG (mg/dL) } \\
\text { ESR (minutes) }\end{array}$ & $\begin{array}{l}214.9 \pm 49.9 \\
141.8 \pm 58.5 \\
48.6 \pm 10.5 \\
132.5 \pm 50.9 \\
144.3 \pm 69.3 \\
54.3 \pm 21.8\end{array}$ & \begin{tabular}{|l|}
$199 \pm 48.4$ \\
$112 \pm 28.2$ \\
$44 \pm 8.8$ \\
$115 \pm 60.3$ \\
$111.7 \pm 14.2$ \\
$70.0 \pm 11.9$
\end{tabular} \\
\hline
\end{tabular}

Abbreviations: ESR, erythrocyte sedimentation rate; HDL-C, high-density lipoprotein cholesterol; LDL-C, low-density lipoprotein cholesterol; mRS, modified Rankin Scale; RBG, random blood glucose; SSS, Scandinavian stroke scale; TC, total cholesterol; TG, triglycerides.

Based on the ROC curve, the optimal cutoff value of serum neopterin levels as an indicator for prediction of poor functional outcome and mortality was projected to be $>16.5$ $\mathrm{nmol} / \mathrm{L}$, which yielded a sensitivity of $100 \%$ and a specificity of $94.7 \%$, with AUC of $0.75(P<0.001)$. Likewise, the optimal cutoff value of serum $\mathrm{SCD} 40 \mathrm{~L}$ levels as an indicator for prediction of poor functional outcome and mortality was projected to be $>8.5 \mathrm{ng} / \mathrm{mL}$, which yielded a sensitivity of $100 \%$ and a specificity of $94.7 \%$, with AUC of $0.83(P<0.001)$.

Table 2 The mean levels of inflammatory markers in all patients with ischemic stroke compared to controls

\begin{tabular}{l|l|l|l}
\hline \multirow{2}{*}{ Parameters } & $\begin{array}{l}\text { Patients } \\
(\mathbf{n}=\mathbf{1 0 0})\end{array}$ & $\begin{array}{l}\text { Controls } \\
(\mathbf{n}=\mathbf{2 5})\end{array}$ & \multirow{2}{*}{-value* } \\
\cline { 2 - 3 } & Mean \pm SD & \\
\hline CRP $(\mathrm{mg} / \mathrm{dL})$ & $4.8 \pm 1.6$ & $2.7 \pm 1.7$ & 0.001 \\
\hline Neopterin $(\mathrm{nmol} / \mathrm{L})$ & $16.04 \pm 3.8$ & $1.3 \pm 0.2$ & 0.001 \\
\hline sCD40 ligand $(\mathrm{ng} / \mathrm{mL})$ & $8.2 \pm 1.9$ & $0.8 \pm 0.6$ & 0.001 \\
\hline
\end{tabular}

Note: *Student's $t$-test was used to compare the mean difference between groups. Abbreviations: CRP, C-reactive protein; sCD40, soluble CD40.
Also, the optimal cutoff value of serum CRP levels as an indicator for prediction of poor functional outcome and mortality was projected to be $>2.5 \mathrm{mg} / \mathrm{dL}$, which yielded a sensitivity of $100 \%$ and a specificity of $63.2 \%$, with AUC of $0.85(P<0.001)$, as shown in Table 5 and Figure $3 \mathrm{~A}-\mathrm{C}$.

Our study reported a non-significant negative correlation between neopterin and SSS $(r=-0.126, P=0.21)$; however, SCD40L and CRP levels had statistically significant negative correlation with SSS $(r=-0.285$ and $-0.252, P<0.05$, respectively). Notably, neopterin, $\mathrm{SCD} 40 \mathrm{~L}$, and CRP levels had statistically significant positive correlations with functional outcome at 90 days (after the onset of stroke) when measured by mRS $(r=0.278,0.490,0.402$, respectively, $P<0.05$ ). A statistically significant positive correlation between neopterin and SCD40L levels was found ( $r=0.425$, $P<0.001)$. CRP level was statistically and significantly correlated positively with both serum neopterin and $\mathrm{SCD} 40 \mathrm{~L}$ levels ( $r=0.330$ and $r=0.171$, respectively, with $P<0.001$ for each). Additionally, there were statistically significant positive correlations between the neopterin level and the 
Table 3 Distribution of serum neopterin and sCD40 ligand levels in stroke patients with favorable and unfavorable outcomes (90 days later)

\begin{tabular}{l|l|l|l}
\hline \multirow{2}{*}{ Parameters } & \multicolumn{2}{|l|}{ mRS (90 days later) $(\mathbf{n}=\mathbf{1 0 0})$} & $\boldsymbol{P}_{\text {-value }}$ \\
\cline { 2 - 3 } & Favorable $(\mathbf{n}=\mathbf{4})$ & Unfavorable $(\mathbf{n}=\mathbf{9 6})$ & \\
\hline Neopterin (mean \pm SD) & $16 \pm 3.6$ & $16.1 \pm 3.8$ & 0.7 \\
\hline sCD40 ligand (mean \pm SD) & $8 \pm 0.6$ & $8.2 \pm 2.1$ & 0.3 \\
\hline
\end{tabular}

Note: 'Student's $t$-test was used to compare the mean difference between groups. Abbreviations: mRS, modified Rankin Scale; sCD40, soluble CD40.

SCD40L level in patients with small artery disease as etiology for AIS ( $P=0.04$ and $P=0.03$, respectively) as shown in Table 6 and Figure 4A-C.

There was also a significant positive correlation between the neopterin level and diabetes mellitus $(r=0.046, P=0.03)$, with no significant correlations with other risk factors. Serum level of sCD40L had statistically significant positive correlations with smoking, hypertension, diabetes mellitus, and dyslipidemia ( $r=0.117,0.1,0.185$, and 0.046 , respectively, with $P<0.05$ ), without any significant correlations with ischemic heart disease and non-valvular AF, as summarized in Table 7.

Table 8 shows the multivariate regression analysis of the significant predictors affecting mortality among the AIS cases. After adjusting for age and sex, the final logistic regression model contained two predictors: serum levels of sCD40L and CRP. In other words, with $1 \mathrm{ng} / \mathrm{mL}$ increase in $\mathrm{SCD} 40 \mathrm{~L}$ serum level, there was 2.65 times increase in the probability of dying from AIS (adjusted OR [AOR] =2.65, 95\% CI 1.03-6.81), and this difference was statistically significant $(P<0.05)$. Likewise, with $1 \mathrm{mg} / \mathrm{dL}$ increase in the
CRP level, there was 2.51 times increase in the probability of dying from AIS (AOR =2.65, 95\% CI 1.15-5.51), which was statistically significant $(P<0.05)$.

\section{Discussion}

Immune responses and inflammation play an important role in the pathogenesis of ischemic stroke. ${ }^{2}$ Recently, strategy for treatment of stroke is based on the assumption that administration of pharmaceuticals may ameliorate progressive tissue injury, consequently improving functional outcome. Recent studies have evaluated the therapeutic potential of gene transfer to reduce ischemic brain injury. For example, antiinflammatory peptides that reduce the inflammatory response, anti-oxidants that reduce damage produced by oxygen radicals, and anti-apoptosis agents that reduce neuronal death all potentially have therapeutic roles in protecting against ischemic brain injury. The current study aimed at assessing the neopterin, sCD40L, and hs-CRP levels in patients with AIS at admission and predicting their role in the functional outcome and mortality after 3 months.
A

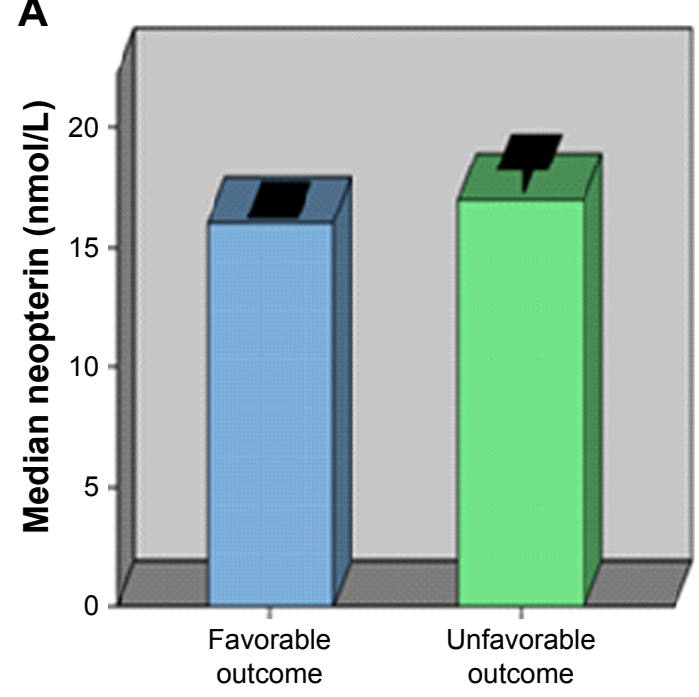

MRS-favorable-unfavorable-outcome

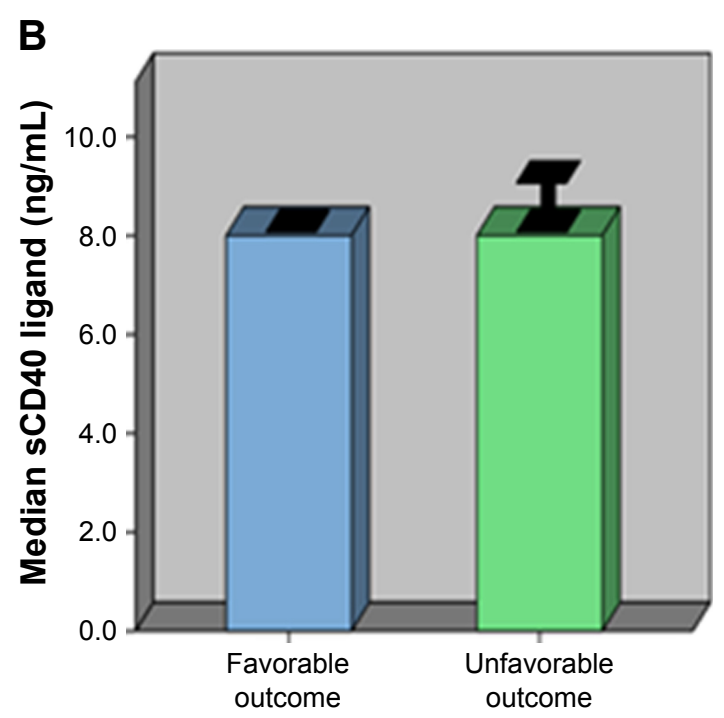

MRS-favorable-unfavorable-outcome

Figure I Mean serum levels of (A) neopterin and (B) sCD40 ligand in acute ischemic stroke patients with favorable and unfavorable outcomes 90 days later. Error bars indicate $95 \% \mathrm{Cl}$ values.

Abbreviation: $\mathrm{sCD} 40$, soluble CD40. 
Table 4 Distribution of serum neopterin and sCD40 Ligand levels according to survival status

\begin{tabular}{l|l|l|l}
\hline Parameters & $\begin{array}{l}\text { Survivors } \\
(\mathbf{n}=\mathbf{7 6})\end{array}$ & $\begin{array}{l}\text { Deaths } \\
(\mathbf{n}=\mathbf{2 4})\end{array}$ & \multirow{2}{*}{-value $^{\mathbf{a}}$} \\
\cline { 2 - 3 } & \multicolumn{2}{|c|}{$($ Mean \pm SD) } & \\
\hline Serum neopterin $(\mathrm{nmol} / \mathrm{L})$ & $16 \pm 3.8$ & $18 \pm 5.2$ & 0.001 \\
\hline Serum $\mathrm{sCD} 40$ ligand $(\mathrm{ng} / \mathrm{mL})$ & $8 \pm 1.35$ & $10 \pm 2.28$ & 0.001 \\
\hline
\end{tabular}

Note: aP-value is significant if $<0.05$.

Abbreviation: $\mathrm{SCD} 40$, soluble CD40.

Our study reported significantly higher levels of neopterin and $\mathrm{SCD} 40 \mathrm{~L}$ in acute stroke patients when compared with controls. This finding was in concordance with previous studies that reported significantly higher serum levels of neopterin and $\mathrm{SCD} 40 \mathrm{~L}$ in patients with AIS when compared with control subjects. ${ }^{21,22}$ Indeed, the increased severity of stroke, as defined by the SSS score, had significant correlation with increasing levels of sCD40L, but not with higher levels of neopterin. These results were in disagreement with many studies which hypothesized that higher levels of neopterin were associated with more severe stroke presentation. However, they found that higher levels of sCD40L in AIS patients were associated with more neurological deficit as assessed by The National Institutes of Health Stroke Scale, reflecting the severity of the stroke. ${ }^{2,15,23}$ This conflicting result could be attributed to methodological differences between the studies; while we evaluated the association between these markers and the severity of stroke at admission only, they determined it at 48 hours after acute IS and after 90 days of follow-up. Moreover, Novo et al and Rosvall et al stated that SCD40L might be a predictive risk factor for cerebrovascular events. ${ }^{24,25}$
In the current study, no significant differences in the levels of neopterin and SCD40L in our patients, with unfavorable functional outcome when compared to those with favorable outcome, were found. Nonetheless, significant correlations between their levels and functional outcomes at 90-day follow-up were observed in our study, indicating prolonged inflammatory response of brain to ischemic injury which in turn is characterized by activation of resident brain cells, production of pro-inflammatory mediators like neopterin, and infiltration of various types of inflammatory cells into ischemic brain tissue. Our findings were in contrast to Lin et al and Zeng et al who stated that neopterin levels were strongly and independently predictive of 90-day unfavorable clinical outcome in patients with AIS. ${ }^{2,23}$ Arsenault et al suggested that plasma levels of neopterin are significantly associated with the risk of poor outcomes in major cardioand cerebrovascular events. ${ }^{26}$ Lai et al found no association between CD40L and stroke outcome. ${ }^{27}$

Moreover, Leonardo reported a higher CD154 expression in platelets of ischemic stroke patients with poor functional outcome, compared with patients with better outcome. ${ }^{28}$ Also, the highest levels of neopterin, sCD40L, and CRP were detected in our deaths, suggesting that they were not only considered as markers for clinical outcome, but could also play a pathogenic role in stroke. These results were in concordance with Zeng et al who found that non-survivor AIS patients had significantly higher neopterin levels than survivors, suggesting that it could be considered as an independent prognostic marker for functional outcome and death in patients with AIS. ${ }^{2}$ Leonardo reported higher serum levels of sCD154
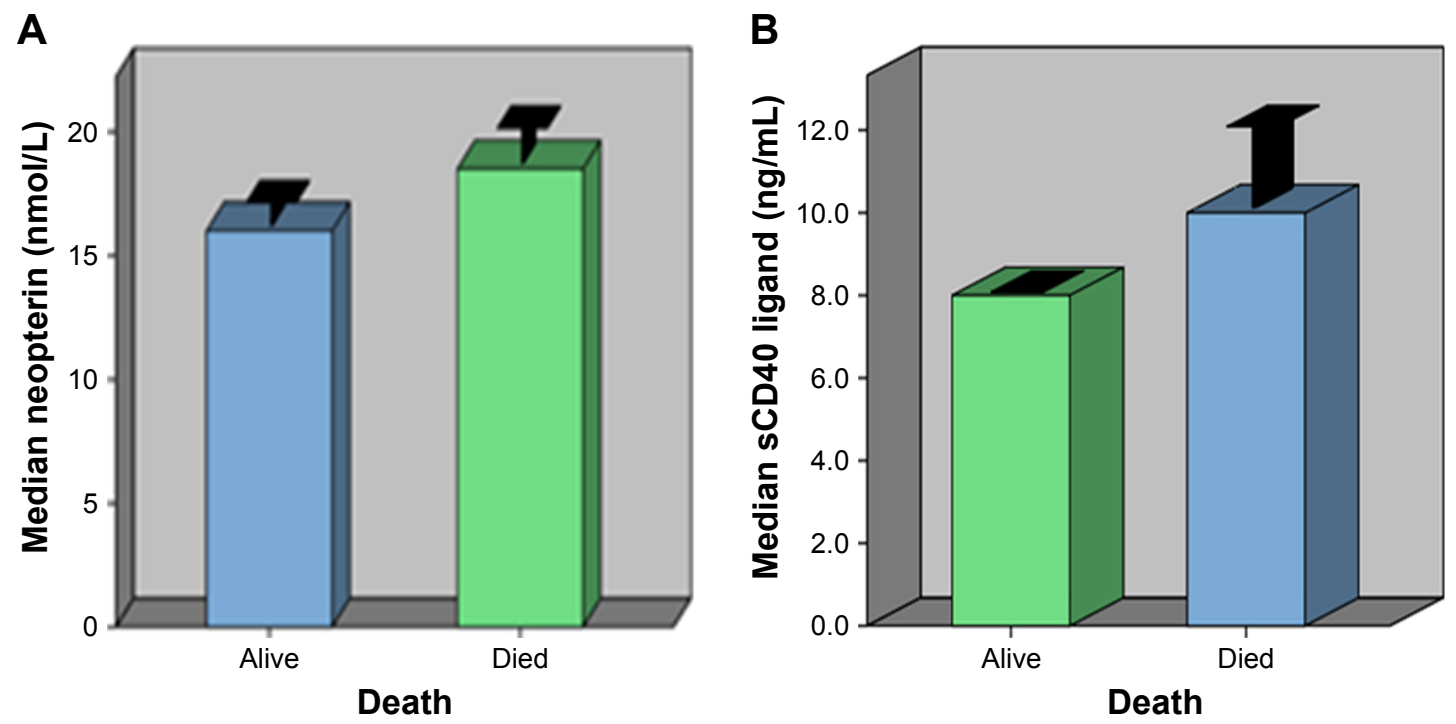

Figure 2 Mean serum levels of (A) neopterin and (B) sCD40 ligand in survivors and those who died with acute ischemic stroke. Error bars indicate $95 \%$ Cl values. Abbreviation: sCD40, soluble CD40. 
Table 5 Prediction of functional outcome and mortality in AIS patients

\begin{tabular}{l|l|l|l}
\hline Parameter & Neopterin & sCD40 ligand & CRP \\
\hline AUC & $75.4 \%$ & $83.3 \%$ & $85.1 \%$ \\
\hline $95 \% \mathrm{Cl}$ & $0.65-0.85$ & $0.70-0.96$ & $0.76-0.94$ \\
\hline Cutoff & $>16.5 \mathrm{nmol} / \mathrm{L}$ & $>8.5 \mathrm{ng} / \mathrm{mL}$ & $>2.5 \mathrm{mg} / \mathrm{dL}$ \\
\hline$P$-value & 0.001 & 0.001 & 0.001 \\
\hline Sensitivity & $100 \%$ & $100 \%$ & $100 \%$ \\
\hline Specificity & $94.7 \%$ & $94.7 \%$ & $63.2 \%$ \\
\hline
\end{tabular}

Abbreviations: AIS, acute ischemic stroke; AUC, area under the curve; CRP C-reactive protein; sCD40, soluble CD40.

A

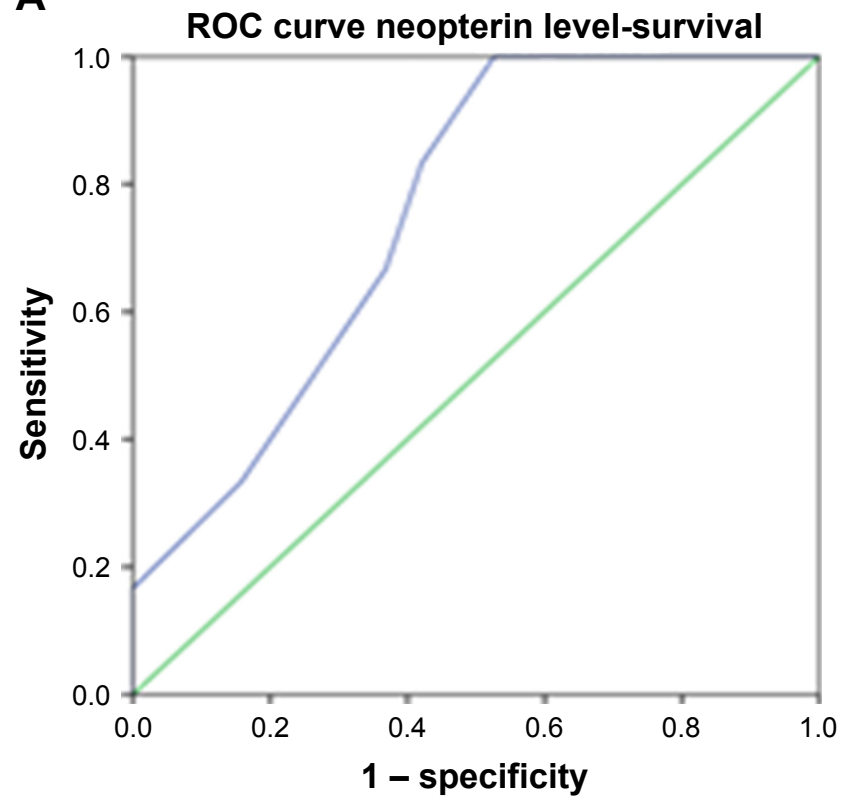

in non-surviving patients than in surviving patients, and an association between serum levels of sCD154 and patient mortality. ${ }^{28}$ They explained this association by a higher inflammatory state and a higher pro-thrombotic state, which could facilitate higher brain ischemia and higher death risk for patients with higher circulating sCD154 levels. Also, Zacho et al reported that as a result of the inflammation that participates in the mechanisms of cerebral injury, high CRP levels could be a sign of an inflammatory state and the increased CRP levels are consistently associated with atherosclerosis and

C

B
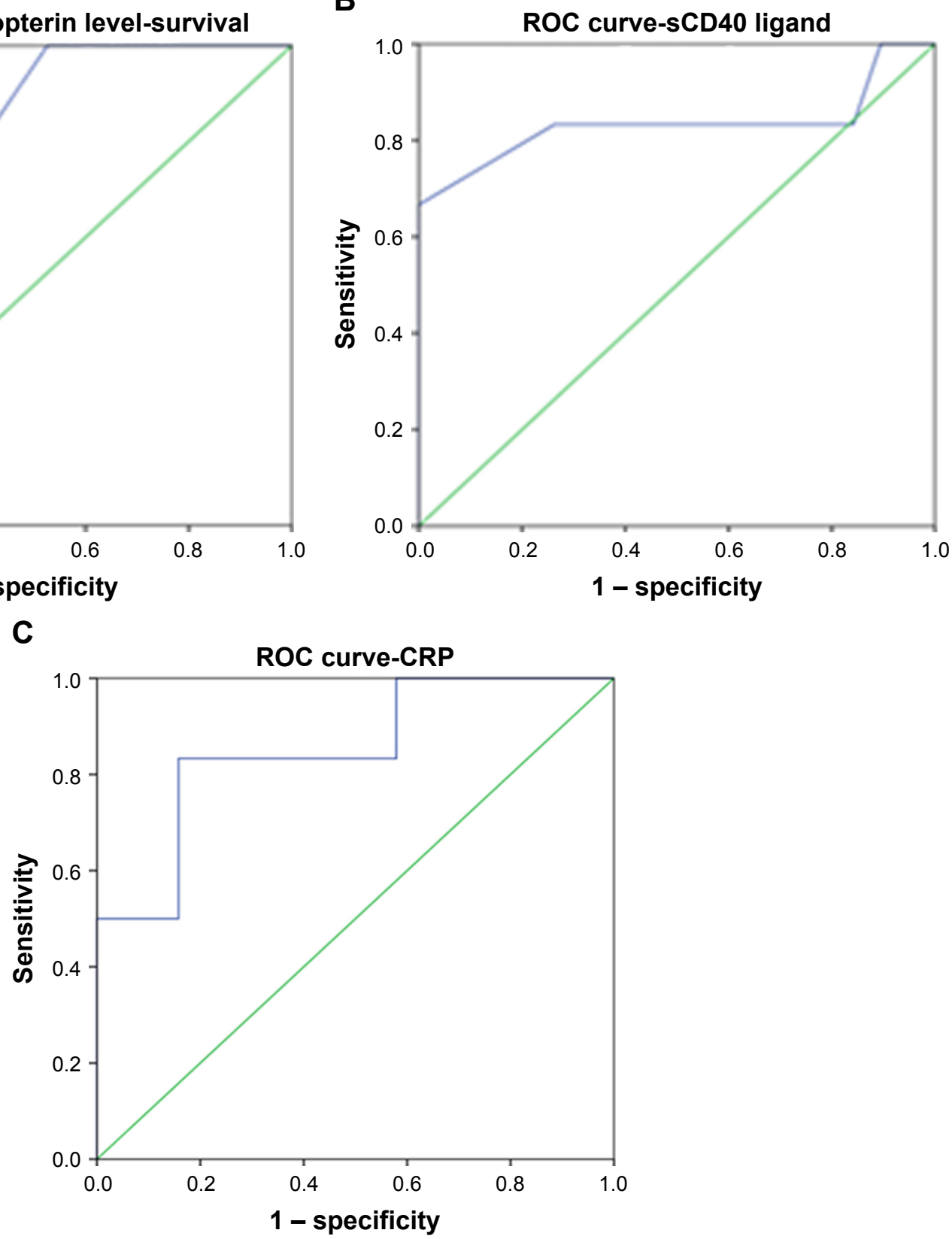

Figure 3 The ROC curve showed the optimal cutoff value of serum (A) neopterin levels, (B) sCD40 ligand levels, and (C) CRP levels as an indicator for prediction of poor functional outcome and mortality.

Abbreviations: CRP, C-reactive protein; ROC, receiver operating characteristic; sCD40, soluble CD40. 
Table 6 Correlation of the serum levels of neopterin, sCD40 ligand, and CRP with the stroke severity and the functional outcome in patients with ischemic stroke

\begin{tabular}{|c|c|c|c|c|c|c|c|c|}
\hline \multirow[t]{2}{*}{ Parameters } & \multicolumn{2}{|l|}{ mRS } & \multicolumn{2}{|c|}{ Neopterin } & \multicolumn{2}{|c|}{ sCD40 ligand } & \multicolumn{2}{|l|}{ CRP } \\
\hline & $r^{a}$ & $P$-value & $r$ & $P$-value & $r$ & $P$-value & $r$ & $P$-value \\
\hline SSS & & & -0.126 & 0.211 & -0.285 & 0.004 & -0.252 & 0.012 \\
\hline Neoptrin & 0.278 & 0.005 & & & 0.425 & 0.001 & 0.330 & 0.001 \\
\hline sCD40 ligand & 0.490 & 0.001 & 0.425 & 0.001 & & & 0.171 & 0.001 \\
\hline CRP & 0.402 & 0.001 & 0.330 & 0.001 & 0.171 & 0.001 & & \\
\hline
\end{tabular}

Note: ${ }^{\text {a }}=$ correlation coefficient.

Abbreviations: CRP, C-reactive protein; mRS, modified Rankin Scale; sCD40, soluble CD40; SSS, Scandinavian Stroke Scale.

vascular disease, suggesting that their elevated concentrations could predict poor survival independently of stroke severity. ${ }^{29}$

Inflammation could accentuate platelet activation and cytokines released by the activated platelets that could induce inflammatory responses. Both inflammation and platelet activation play important roles in acute ischemic vascular events. ${ }^{29}$ Aloui et al and Thomas and Storey reported that platelet activation plays a key role in forming
A

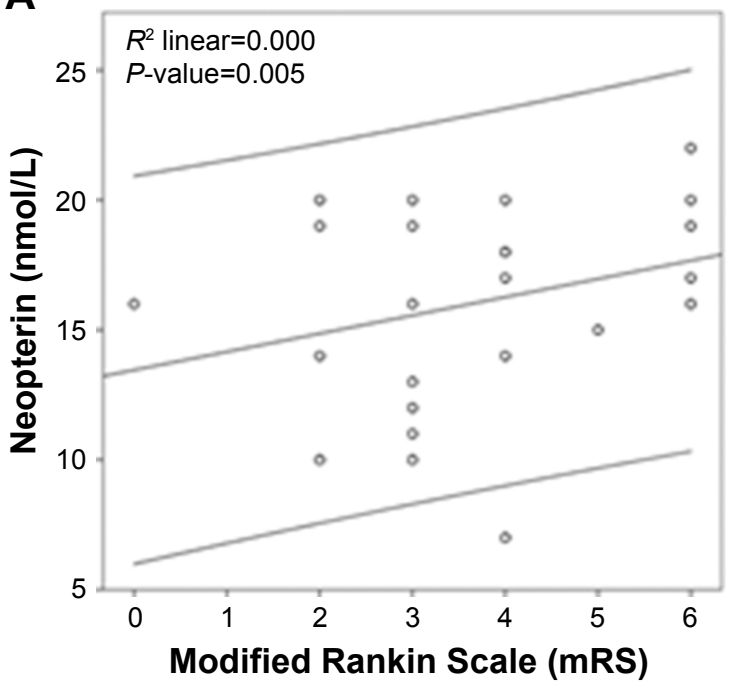

B

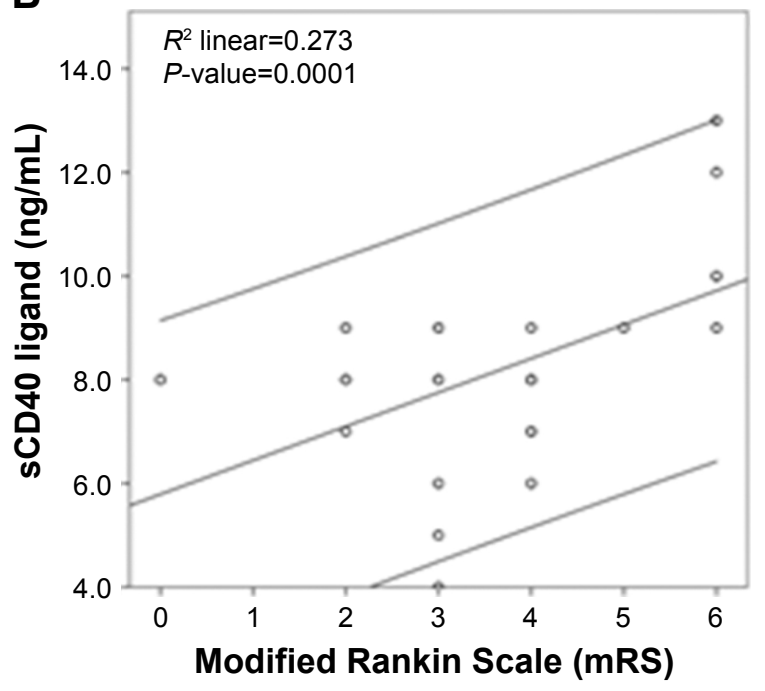

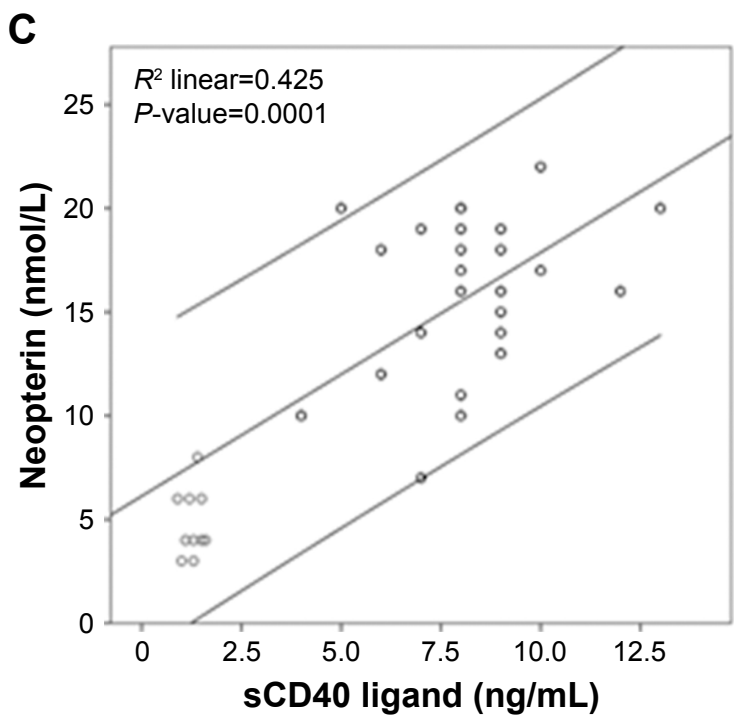

Figure 4 Correlation between mean serum levels of (A) neopterin and (B) sCD40 ligand with functional outcome assessed by modified Rankin Scale (mRS), and (C) correlation between mean serum levels of neopterin and sCD40 ligand.

Abbreviation: sCD40, soluble CD40. 
Table 7 Correlation of the serum levels of neopterin and sCD40 ligand with the risk factors of stroke in patients with ischemic stroke

\begin{tabular}{l|l|l|l|l}
\hline \multirow{2}{*}{ Risk factors } & \multicolumn{2}{l}{ Neopterin } & \multicolumn{2}{l}{ SCD40 ligand } \\
\cline { 2 - 5 } & $\boldsymbol{R}$ & $\boldsymbol{P}$-value & $\boldsymbol{r}$ & $\boldsymbol{P}$-value \\
\hline Hypertension & 0.006 & 0.49 & 0.117 & $\mathbf{0 . 0 0 1}$ \\
\hline Diabetes mellitus & 0.046 & $\mathbf{0 . 0 3 3}$ & 0.1 & $\mathbf{0 . 0 0 1}$ \\
\hline Dyslipidemia & 0.003 & 0.574 & 0.185 & $\mathbf{0 . 0 0 1}$ \\
\hline Smoking & 0.010 & 0.322 & 0.046 & $\mathbf{0 . 0 3 2}$ \\
\hline Ischemic heart disease & 0.026 & 0.112 & 6.734 & 0.798 \\
\hline Atrial fibrillation & 0.032 & 0.075 & 1.323 & 0.91 \\
\hline Abnormal carotid duplex & 0.071 & $\mathbf{0 . 0 0 7}$ & 0.089 & $\mathbf{0 . 0 0 3}$ \\
\hline Abnormal vertebrobasilar duplex & 0.093 & $\mathbf{0 . 0 4 9}$ & 0.025 & 0.798 \\
\hline
\end{tabular}

Notes: $R=$ correlation coefficient. Bold values indicate statistical significance.

Abbreviation: $\mathrm{sCD} 40$, soluble CD40.

and extending atherosclerotic plaques. These activated platelets release $\mathrm{SCD} 40 \mathrm{~L}$-inducing platelet aggregation and platelet-leukocyte conjugation that may lead to atherothrombosis and inflammation which is well known to play significant roles in the occurrence and the development of atherosclerosis..$^{30,31}$

Nevertheless, Hermus et al stated that increased inflammation has been reported in patients with carotid artery disease and AIS. ${ }^{32}$ In our study, significant associations between the highest mean levels of neopterin and sCD40L with atherosclerotic changes and carotid stenosis were found. Our results were in agreement with Chen et al who reported that higher levels of circulating neopterin were found in patients with severe carotid artery stenosis and AIS. ${ }^{33}$ Notably, Hermus et al and Sugioka et al had reported that the neopterin levels were higher in patients with carotid artery disease than in healthy subjects. ${ }^{32,34}$ Also, Wang et al and Ding et al stated that the plasma sCD40L levels of patients with ischemic stroke and carotid plaques were significantly higher than those of asymptomatic patients, suggesting that an elevated plasma sCD40L level plays an important role in

Table 8 Multivariate logistic regression analysis for determining the role of neopterin, SCD40 ligand, and CRP in predicting mortality in our patients with acute ischemic stroke

\begin{tabular}{l|l|l|l}
\hline Variables & AOR & $\mathbf{9 5 \%} \mathbf{C I}$ & $\mathbf{P}$-value \\
\hline Age/years & 1.13 & $0.99-I .28$ & 0.068 \\
\hline Sex (female) & 1.80 & $0.62-6.35$ & 0.531 \\
\hline Neopterin & 1.53 & $0.98-2.39$ & 0.062 \\
\hline SCD40L & $\mathbf{2 . 6 5}$ & $\mathbf{I . 0 3 - 6 . 8 I}$ & $\mathbf{0 . 0 4 3}$ \\
\hline CRP & $\mathbf{2 . 5 I}$ & $\mathbf{I . 1 5 - 5 . 5 I}$ & $\mathbf{0 . 0 2 2}$ \\
\hline
\end{tabular}

Notes: The model included age and sex as a priori factors in addition to the significant factors in the univariate analysis. Bold values indicate statistical significance. Abbreviations: AOR, adjusted odds ratio; CRP, C-reactive protein; SCD40L, soluble CD40 ligand. platelet activation and arteriosclerotic thrombosis formation in patients with ischemic stroke. ${ }^{17,35}$

Furthermore, in the current study, more elevated CRP levels were observed in our stroke patients compared to controls. Elevated levels of CRP were correlated with stroke severity and poor functional outcome in our patients, where patients with elevated CRP had lower SSS and higher mRS scores. However, our patients still had normal levels of CRP after stroke, implying that ischemic stroke itself does not induce a full-blown acute phase response. These findings were in concordance with Chen et al who reported that CRP is increased during both acute and convalescent phases of ischemic stroke and is independently predictive of 90-day major adverse neurological events in patients with ischemic stroke. ${ }^{33}$

Moreover, Elkind et al stated that CRP measured predominantly within the first 72 hours post-stroke was associated with an increased risk of mortality over the following 5 years, after adjusting for confounding factors. ${ }^{20}$ Song et al reported that higher plasma concentration of CRP was associated with a worse neurologic outcome. ${ }^{36}$ Ferro et al showed that a high CRP level at admission is associated with more severe stroke, cardioembolic etiology, poor short-term functional outcome, and higher mortality. ${ }^{37}$ Therefore, we considered that increased CRP level following stroke was not only a consequence of brain infarction but also contributes to ischemic damage as well.

In the current study, we found significant positive correlations between neopterin, $\mathrm{SCD} 40 \mathrm{~L}$, and CRP levels. These results were in agreement with Zeng et al. ${ }^{2}$ However, Ferro et al, Heeschen et al, and de Lemos et al reported no relationship between sCD40L and CRP levels, indicating that they likely represent two different pathways of the inflammatory status. ${ }^{37-39}$ Therefore, these inflammatory biomarkers are 
potentially mutual factors predictive of prognostic outcomes in patients with AIS.

In contrast to Ferro et $\mathrm{al}^{37}$ who reported that patients with non-valvular AF and with higher SCD40L levels were at higher risk of ischemic stroke, we observed no correlations between the highest levels of $\mathrm{sCD} 40 \mathrm{~L}$, serum neopterin, ischemic heart diseases, and AF in our AIS patients. Moreover, El-Shahhat et al found higher CD40L levels in patients with unstable angina, reflecting the important pathogenic aspects in these patients. ${ }^{40}$

We found that serum neopterin level was positively correlated only with the presence of diabetes mellitus as risk factor for AIS. This result was in agreement with Grossmann et al who showed a weakly positive association between neopterin and diabetes ${ }^{41}$ Increased neopterin levels in diabetic patients could be explained by enhanced granulocyte aggregation in subjects with diabetes influenced by the intense metabolic disturbances. Activated granulocytes can also trigger vascular damage, which might contribute to the development of atherosclerosis. However, there were no correlations between neopterin and other studied risk factors of AIS in our study. These findings were in contrast with Schennach et al who reported a weak, but highly significant, correlation of neopterin concentrations with DBP, owing to the fact that atherosclerosis, which is associated with immune activation and higher neopterin production, might possibly lead to vasoconstriction and thereby increased DBP. ${ }^{42}$ Also, they reported that there were non-significant correlations of higher neopterin levels with serum cholesterol and SBP. Notably, Schennach et al found that smokers showed lower levels of neopterin, clarifying the suppressive effect of tobacco smoke on the human immune system. ${ }^{42}$

Hypercholesterolemia and type 2 diabetes mellitus (T2DM) are two major risk factors for atherosclerosis development and progression, and both are often associated with a hypercoagulable state, an increased platelet adhesion and aggregation, and a state of subclinical inflammation. Markers of vascular inflammation have emerged as new independent predictors of cardiovascular events, eg, CD40L ${ }^{43}$ In the current study, we found positive correlations of higher levels of SCD40L with diabetes, hypertension, dyslipidemia, and smoking. These results agreed with Davi et al who stated that diabetic patients with AIS had higher plasma SCD40L than controls, because T2DM is characterized by persistent platelet activation, which is responsible for the increased plasma levels of sCD40L. ${ }^{44}$ Seijkens et al demonstrated the involvement of the CD40-CD40L axis in the development and progression of T2DM and its vascular complications and suggested that inhibition of CD40-CD40L may reduce the systemic inflammatory response responsible for the progression of T2DM and the development of cardiovascular disease (CVD) ${ }^{45}$ Moreover, Lievens et al and Vaidyula et al reported that both hyperglycemia and chronic hyperinsulinemia increase the expression of membrane-bound CD40L on circulating platelets, which subsequently promotes the formation of platelet-leukocyte aggregates and leukocyte-endothelium interactions, thereby enhancing vascular inflammation. ${ }^{46,47}$ Harding et al and Levitzky et al stated that the expression of CD40L on platelets and platelet-monocyte aggregates significantly increased in healthy smokers compared to non-smokers. ${ }^{48,49}$ This could be explained by the fact that platelets increased their activation as a response to cigarette smoke with release of sCD40L from activated platelets. The release of $\mathrm{sCD} 40 \mathrm{~L}$ from platelets upon activation indicates the hypercoagulable state that promotes vascular thrombosis in active smokers. ${ }^{50}$

In contrast to our results, Lim et al did not find any significant correlations between $\mathrm{SCD} 40 \mathrm{~L}$ and total cholesterol, glycemia, and blood pressure, suggesting alternative mechanism(s) for the elevated sCD40L and abnormal platelet activation in patients. ${ }^{51}$ Moreover, Jefferis et al stated that serum $\mathrm{SCD} 40 \mathrm{~L}$ levels were associated with cigarette smoking status, but were not consistently associated with classic cardiovascular risk factors (lipids and blood pressure). ${ }^{52}$

In the current study, significantly higher levels of neopterin and $\mathrm{SCD} 40 \mathrm{~L}$ were found in AIS patients with small artery disease as an etiology for stroke. Our finding was in concordance with that reported by Rouhl et al who demonstrated that neopterin levels were higher in lacunar stroke patients than in hypertension patients, suggesting that activated monocytes/macrophages play a role in the pathophysiology of CVD. ${ }^{53}$ Similarly, Oberheiden et al revealed a significantly higher platelet surface expression of CD40L in patients with lacunar stroke (based on small artery disease) than in patients with territorial stroke (based on large artery disease). ${ }^{54}$ However, in disagreement with our results, Davi et al stated that there were no significant differences in plasma CD40L among different TOAST groups indicating that CD40-CD40L activation may be one of the universally active mechanisms shortly before or after ischemia. ${ }^{44}$ Likewise, Tsai et al reported higher plasma levels of CD40L in patients with large artery disease stroke, significantly differing from patients with cardioembolic stroke. ${ }^{22}$ Wang et al stated that CD40L, on peripheral blood monocytes, also was higher in patients with the large-artery atherosclerosis stroke type than in patients with any other type of acute cerebral infarction 
or pure carotid artery stenosis, suggesting that circulating monocytes with high CD40L expression may be associated with cerebral atherosclerosis and plaque instability. ${ }^{14}$

The current study encountered some limitations. First, the study evaluated the short-term outcome (90-day follow-up). The relationship between long-term outcome and neopterin, sCD40L, and CRP has not yet been studied. The second issue was non-randomized sampling technique, which may affect the generalizability of the results.

\section{Conclusion}

The evaluated serum levels of neopterin, sCD40L, and CRP have been associated with unfavorable 90-day stroke outcome and have been predictive of death in patients with AIS.

\section{Recommendations}

Future research work should be conducted to assess the level of inflammatory markers as predictors of patient neurological deficit, outcome, or follow-up. Disease progression and treatment efficacy is a new era for the modulation of treatment. Development of antibodies against these involved inflammatory markers is a new line of treatment for amelioration of post-stroke outcome, and this could be a promising way to prevent deterioration in AIS.

\section{Disclosure}

The authors report no conflicts of interest in this work.

\section{References}

1. Elali A, Jean LeBlanc N. The role of monocytes in ischemic stroke pathobiology: new avenues to explore. Front Aging Neurosci. 2016;8:29.

2. Zeng X, Zhang G, Yang B, et al. Neopterin as a predictor of functional outcome and mortality in Chinese patients with acute ischemic stroke. Mol Neurobiol. 2016;53(6):3939-3947.

3. Jin R, Liu L, Zhang S, Nanda A, Li G. Role of inflammation and its mediators in acute ischemic stroke. J Cardiovasc Transl Res. 2013;6(5): 834-851.

4. Jin R, Yang G, Li G. Inflammatory mechanisms in ischemic stroke: role of inflammatory cells. J Leukoc Biol. 2010;87(5):779-789.

5. Whiteley W, Wardlaw J, Dennis M, et al. The use of blood biomarkers to predict poor outcome after acute transient ischemic attack or ischemic stroke. Stroke. 2012;43(1):86-91.

6. Markaki I, Franzén I, Talani C, Loizou L, Kostulas N. Long-term survival of ischemic cerebrovascular disease in the acute inflammatory stroke study, a hospital-based cohort described by TOAST and ASCO. Cerebrovasc Dis. 2013;35(3):213-219.

7. Celik C, Erdem M, Caycı T, et al. The association between serum levels of neopterin and number of depressive episodes of major depression. Prog Neuropsychopharmacol Biol Psychiatry. 2010;34(2):372-375.

8. Murr C, Widner B, Wirleitner B, Fuchs D. Neopterin as a marker for immune system activation. Curr Drug Metab. 2002;3(2):175-187.

9. Parker DC, Mielke MM, Yu Q, et al. Plasma neopterin level as a marker of peripheral immune activation in amnestic mild cognitive impairment and Alzheimer's disease. Int J Geriatr Psychiatry. 2013;28(2): 149-154.
10. Millner MM, Franthal W, Thalhammer GH, et al. Neopterin concentrations in cerebrospinal fluid and serum as an aid in differentiating central nervous system and peripheral infections in children. Clin Chem. 1998; 44(1):161-167.

11. Zeimet AG, Widschwendter M, Knabbe C, et al. Ascitic interleukin-12 is an independent prognostic factor in ovarian cancer. J Clin Oncol. 1998;16(5):1861-1868.

12. Oda K, Arai T, Nagase M. Increased serum and urinary neopterin in nephrotic syndrome indicate cell-mediated immune dysfunction. Am J Kidney Dis. 1999;34(4):611-617.

13. VanGilder RL, Davidov DM, Stinehart KR, et al. C-reactive protein and long term ischemic stroke pro-gnosis. J ClinNeurosci. 2014; 21(4):547-553.

14. Wang JH, Zhang YW, Zhang P, et al. CD40 ligand as a potential biomarker for atherosclerotic instability. Neurol Res. 2013;35(7): 693-700.

15. Wang K, Wang J, Pan X, et al. Association of plasma soluble CD40L and P-selectin with large-artery atherosclerosis stroke. Int J Clin Exp Pathol. 2017;10(4):4827-4832.

16. Antoniades C, Bakogiannis C, Tousoulis D, Antonopoulos AS, Stefanadis C. The CD40/CD40 ligand system: linking inflammation with atherothrombosis. J Am Coll Cardiol. 2009;54(8):669-677.

17. Wang YJ, Gong ZQ, Bi XM, Li YL. Correlation of plasma soluble cluster of differentiation 40 ligand, alpha fetoprotein A, and pregnancyassociated plasma protein A with carotid plaque in patients with ischemic stroke. Genet Mol Res. 2015;14(3):8091-8099.

18. Elias-Smale SE, Kardys I, Oudkerk M, Hofman A, Witteman JC. $\mathrm{C}$-reactive protein is related to extent and progression of coronary and extra-coronary atherosclerosis; results from the Rotterdam study. Atherosclerosis. 2007;195(2):e195-e202.

19. Calabrò P, Golia E, Yeh ET. Role of C-reactive protein in acute myocardial infarction and stroke: possible therapeutic approaches. Curr Pharm Biotechnol. 2012;13(1):4-16.

20. Elkind MS, Tai W, Coates K, Paik MC, Sacco RL. High-sensitivity C-reactive protein, lipoprotein-associated phospholipase A2, and outcome after ischemic stroke. Arch Intern Med. 2006;166(19): 2073-2080.

21. Ulvi H, Emre H, Demir R, Aygul R, Varoğlu A, Kara F. Neopterin levels in patients with cerebrovascular disease. Eurasian J Med. 2008; 40(2):79-82.

22. Tsai NW, Chang WN, Shaw CF, et al. Levels and value of platelet activation markers in different subtypes of acute non-cardio-embolic ischemic stroke. Thromb Res. 2009;124(2):213-218.

23. Lin HS, Tsai TH, Liu CF, et al. Serum level and prognostic value of neopterin in patients after ischemic stroke. Clin Biochem. 2012;45(18): 1596-1601.

24. Novo S, Basili S, Tantillo R, et al. Soluble CD40L and cardiovascular risk in asymptomatic low-grade carotid stenosis. Stroke. 2005;36: 673-675.

25. Rosvall M, Janzon L, Berglund G, Engström G, Hedblad B. Incident coronary events and case fatality in relation to common carotid intimamedia thickness. J Intern Med. 2005;257(5):430-437.

26. Arsenault BJ, Barter P, Demicco DA, et al. Prediction of cardiovascular events in statin-treated stable coronary patients of the treating to new targets randomized controlled trial by lipid and non-lipid biomarkers. PLoS One. 2014;9(12):e114519.

27. Lai PT, Chen SY, Lee YS, Ho YP, Chiang YY, Hsu HY. Relationship between acute stroke outcome, aspirin resistance, and humoral factors. J Chin Med Assoc. 2012;75(10):513-518.

28. Leonardo L. New prognostic biomarkers in patients with ischemic stroke. Mol Med Chem. 2015;1:e972.

29. Zacho J, Tybjaerg-Hansen A, Jensen JS, Grande P, Sillesen H, Nordestgaard BG. Genetically elevated C-reactive protein and ischemic vascular disease. N Engl J Med. 2008;359(18):1897-1908.

30. Aloui C, Prigent A, Sut C, et al. The signaling role of CD40 ligand in platelet biology and in platelet component transfusion. Int J Mol Sci. 2014;15(12):22342-22364. 
31. Thomas MR, Storey RF. The role of platelets in inflammation. Thromb Haemost. 2015;114(3):449-458.

32. Hermus L, Schuitemaker JH, Tio RA, et al. Novel serum biomarkers in carotid artery stenosis: useful to identify the vulnerable plaque? Clin Biochem. 2011;44(16):1292-1298.

33. Chen YL, Tsai TH, Sung PH. Levels of circulating neopterin in patients with severe carotid artery stenosis undergoing carotid stenting. J Atheroscler Thromb. 2014;21(2):129-139.

34. Sugioka K, Naruko T, Matsumura Y, et al. Neopterin and atherosclerotic plaque instability in coronary and carotid arteries. $J$ Atheroscler Thromb. 2010;17(11):1115-1121.

35. Ding S, Zhang M, Zhao Y, et al. The role of carotid plaque vulnerability and inflammation in the pathogenesis of acute ischemic stroke. Am J Med Sci. 2008;336(1):27-31.

36. Song IU, Kim JS, Kim YI, Lee KS, Jeong DS, Chung SW. Relationship between high-sensitivity C-reactive protein and clinical functional outcome after acute ischemic stroke in a Korean population. Cerebrovasc Dis. 2009;28(6):545-550.

37. Ferro D, Loffredo L, Polimeni L, et al. Soluble CD40 ligand predicts ischemic stroke and myocardial infarction in patients with nonvalvular atrial fibrillation. Arterioscler Thromb Vasc Biol. 2007;27(12):2763-2768.

38. Heeschen C, Dimmeler S, Hamm CW, et al; CAPTURE Study Investigators. Soluble CD40 ligand in acute coronary syndromes. $N$ Engl J Med. 2003;348(12):1104-1111

39. de Lemos JA, Zirlik A, Schönbeck U, et al. Associations between soluble CD40 ligand, atherosclerosis risk factors, and subclinical atherosclerosis: results from the Dallas Heart Study. Arterioscler Thromb Vasc Biol. 2005;25(10):2192-2196.

40. El-Shahhat N, Ramadan MM, El-Malkey N, Omar AA, Abd El-Aal IA, Eneen A. Soluble CD40 ligand, interleukin (IL)-6, and hemostatic parameters in metabolic syndrome patients with and without overt ischemic heart disease. The Egyptian Heart Journal. 2011;63(3-4):131-135.

41. Grossmann V, Schmitt VH, Zeller T, et al. Profile of the Immune and Inflammatory Response in Individuals With Prediabetes and Type 2 Diabetes. Diabetes Care. 2015;38(7):1356-1364.

42. Schennach H, Murr C, Gächter E, Mayersbach P, Schönitzer D, Fuchs D. Factors influencing serum neopterin concentrations in a population of blood donors. Clin Chem. 2002;48(4):643-645.
43. Santini E, Madec S, Corretti V, Ferrannini E, Solini A. Effect of statins on soluble CD40 ligand in hypercholesterolemic Type 2 diabetic patients. J Endocrinol Invest. 2008;31(7):660-665.

44. Davì G, Tuttolomondo A, Santilli F, et al. CD40 ligand and MCP-1 as predictors of cardiovascular events in diabetic patients with stroke. $J$ Atheroscler Thromb. 2010;16(6):707-713.

45. Seijkens T, Kusters P, Engel D, Lutgens E. CD40-CD40L: linking pancreatic, adipose tissue and vascular inflammation in type 2 diabetes and its complications. Diab Vasc Dis Res. 2013;10(2):115-122.

46. Lievens D, Zernecke A, Seijkens T, et al. Platelet CD40L mediates thrombotic and inflammatory processes in atherosclerosis. Blood. 2010; 116(20):4317-4327.

47. Vaidyula VR, Rao AK, Mozzoli M, Homko C, Cheung P, Boden G. Effects of hyperglycemia and hyperinsulinemia on circulating tissue factor procoagulant activity and platelet CD40 ligand. Diabetes. 2006; 55(1):202-208.

48. Harding SA, Sommerfield AJ, Sarma J, et al. Increased CD40 ligand and platelet-monocyte aggregates in patients with type 1 diabetes mellitus. Atherosclerosis. 2004;176(2):321-325.

49. Levitzky YS, Guo CY, Rong J, et al. Relation of smoking status to a panel of inflammatory markers: the framingham offspring. Atherosclerosis. 2008;201(1):217-224.

50. Kayrak M, Bacaksiz A, Ulgen MS, et al. Plasma concentrations of soluble CD40 ligand in smokers with acute myocardial infarction: a pilot study. Heart Vessels. 2011;26(2):131-137.

51. Lim HS, Blann AD, Lip GY. Soluble CD40 ligand, soluble P-selectin, interleukin-6, and tissue factor in diabetes mellitus: relationships to cardiovascular disease and risk factor intervention. Circulation. 2004; 109(21):2524-2528.

52. Jefferis BJ, Whincup PH, Welsh P, et al. Prospective study of circulating soluble CD40 ligand concentrations and the incidence of cardiovascular disease in a nested prospective case-control study of older men and women. J Thromb Haemost. 2011;9:1452-1459.

53. Rouhl RP, Damoiseaux JG, Lodder J, et al. Vascular inflammation in cerebral small vessel disease. Neurobiol Aging. 2012;33(8):1800-1806.

54. Oberheiden T, Nguyen XD, Fatar M, et al. Platelet and monocyte activation in acute ischemic stroke - is there a correlation with stroke etiology? Clin Appl Thromb Hemost. 2012;18(1):87-91.
Neuropsychiatric Disease and Treatment

\section{Publish your work in this journal}

Neuropsychiatric Disease and Treatment is an international, peerreviewed journal of clinical therapeutics and pharmacology focusing on concise rapid reporting of clinical or pre-clinical studies on a range of neuropsychiatric and neurological disorders. This journal is indexed on PubMed Central, the 'PsycINFO' database and CAS,
Dovepress

and is the official journal of The International Neuropsychiatric Association (INA). The manuscript management system is completely online and includes a very quick and fair peer-review system, which is all easy to use. Visit http://www.dovepress.com/testimonials.php to read real quotes from published authors. 